\title{
Pacific
}

Journal of

Mathematics

\section{MINIMAL HYPERSPHERES IN TWO-POINT HOMOGENEOUS SPACES}

\section{PER TOMTER}




\title{
MINIMAL HYPERSPHERES IN TWO-POINT HOMOGENEOUS SPACES
}

\author{
PER TOMTER
}

\begin{abstract}
In Riemannian geometry the study of minimal submanifolds has given the most important, higher-dimensional generalizations of geodesics. Especially significant from a global point of view are closed minimal submanifolds (generalizing closed geodesics); these raise many hard problems. In this paper we study existence and uniqueness questions in the case of the simplest topological type; i.e. minimal hyperspheres. We restrict ourselves to study such questions for the compact two-point homogeneous spaces; these spaces constitute the most natural generalization of classical (three-point homogeneous) spherical geometry. They can be characterized equivalently as (i) compact two-point homogeneous spaces, (ii) compact rank 1 symmetric spaces, or (iii) irreducible compact positively curved symmetric spaces. Since the standard spheres have been investigated in great detail in connection with the "Spherical Bernstein Problem", we only consider the complex projective spaces $\mathrm{C} P(n)$, the quaternionic projective spaces $H P(n)$, and the Cayley projective plane $C a(2)$ here.
\end{abstract}

\section{Introduction.}

The compact two-point homogeneous spaces are also precisely the symmetric spaces which have a homogeneous, minimal hypersphere, unique up to congruence, and determined as the principal isotropy group orbit of maximal volume. We call it the "equator" of the space. Hence this is the natural class of spaces to study uniqueness questions analogous to the Bernstein problem; i.e. the following question: Is any minimal hypersphere in a compact twopoint homogeneous space an equator?

The most effective method has been the "equivariant differential geometry" initiated by Wu-Yi Hsiang and B. Lawson. In the case of a cohomogeneity two subgroup $G$ of the isometry group the reduced minimal equation. in the orbit space is the geodesic equation for a suitably chosen metric; and the problem of finding $G$-invariant closed minimal submanifolds is reduced to finding geodesics that may "close up" by satisfying a delicate intersection requirement with the singular boundary. The technical difficulties in proving 
the existence of such "closed geodesics" may be expected to depend strongly on the coefficients of the reduced minimal equation; this is born out very clearly in the increasing difficulties in proving the following results:

Theorem 1. There exist infinitely many congruence classes of imbedded, minimal hyperspheres in $\mathbf{C P}(n) ; n \geq 2$.

Theorem 2. There exist infinitely many congruence classes of immersed, minimal hyperspheres in $\mathbf{H} P(n) ; n \geq 1$.

Theorem 3. There exist infinitely many congruence classes of immersed, minimal hyperspheres in the Cayley projective plane.

In fact, we will obtain a very explicit expression for how the oscillatory behaviour of a second order linearized variational equation for the reduced minimal equation changes with the dimension of the scalar field $(\mathbf{C}, \mathbf{H}$ or $\mathrm{Ca}$ ). Thus, although these spaces all have rich two-point homogeneous symmetry, the subtle changes in those symmetry groups related to change of scalars strongly impact existence proofs for non-equatorial minimal hyperspheres.

Theorem 1 was proved in Hsiang, W.T., Hsiang, W.Y. and Tomter, P.: "On the existence of minimal hyperspheres in compact symmetric spaces". Ann. Scient. Ec. Norm. Sup., Vol. 21,(1988), 287-305. In that case the proof is much simplified by lifting in the Hopf fibration $S^{1} \rightarrow S^{2 n+1} \rightarrow$ $\mathbf{C P}(n)$; the orbital invariants are then easier to compute. This belongs to the simplest class of equivariant geometric structures ("corner singularity of focal type"), which enables one to prove Theorem 1 without too much analytical complication. The quaternionic projective space can also be treated by lifting in the Hopf fibration $S^{3} \rightarrow S^{4 n+3} \rightarrow \mathbf{H} P(n)$, the resulting dynamical system is not of the above simple class and requires more complicated analysis in the orbit space ("corner singularity of nodal type"). It should, however, be feasible to compute the orbital invariants directly in the projective spaces; although harder, this more general approach should be of interest for other symmetric spaces. It becomes imperative in the case of the Cayley projective plane, since it is well known that there cannot exist any corresponding "Hopf fibration" $S^{7} \rightarrow S^{23} \rightarrow C a(2)$.

We therefore choose to give a unified treatment for direct computation of the orbital invariants for all these symmetric spaces. This is done in explicit detail for $C a(2)$, which is the more complicated case, involving aspects (triality) of exceptional Lie groups. 


\section{Preliminary results.}

We first observe that only the above cases are relevant for the study of closed, minimal hyperspheres in two-point homogeneous spaces:

Proposition 1. Let $M$ be a Riemannian two-point homogeneous space and let $N$ be a minimal hypersphere in $M$. Then $M$ is either a sphere, a complex or quaternionic projective space, or the Cayley projective plane.

Proof. As is well-known, a simply connected Riemannian manifold of nonpositive curvature is diffeomorphic to Euclidean space and has no compact, minimal submanifolds. Since any two-point homogeneous space of nonpositive sectional curvature is simply connected, we only need to consider those of positive curvature. By Frankel's theorem $([\mathbf{F}])$ it follows that for any closed minimal hypersurface $N$ in a Riemannian manifold $M$ of positive Ricci curvature, the induced map between fundamental groups is surjective: This excludes the real projective spaces, and the result follows from the classification of two-point homogeneous spaces.

Proposition 2. Let $G$ be a cohomogeneity two Lie group of isometrics acting on the Riemannian manifold $M$ and let $\pi: M \rightarrow M / G=X$ be the projection onto the orbit space. Let $M^{*}$ be the union of principal orbits in $M$, and let $X^{*}=\pi\left(M^{*}\right)$, with its orbital distance metric. If $\gamma$ is a curve in $X^{*}$, the mean curvature of $\pi^{-1}(\gamma)$ in $M^{*}$ is given by: $H(z)=$ $k(\pi(z))-\frac{d}{d n} \ln V(\pi(z))$, where $k$ is the geodesic curvature of $\gamma, n$ is the unit normal of $\gamma$, and $V$ is the volume functional registering the volume of the orbits.

This standard result makes it clear that the only needed data from the orbital geometry are the orbital distance metric of $X^{*}$ and the volume functional $V$.

Note: We always allow normalization of $V$ by a multiplicative constant; this does not change the equation.

Now, let $M=\mathbf{C} P(n), \mathbf{H} P(n)$, or $C a(2)$, and let $p \in M$. Let $G$ be the isometry-isotropy group of $p$; by two-point homogeneity the principal orbits of $G$ are hyperspheres. The cut locus $C(p)$ is a $\mathbf{C} P(n-1), \mathbf{H} P(n-1)$, or $C a(1)=S^{8}$ respectively. We normalize the Riemannian metric such that the injectivity radius is $\frac{\pi}{2}$, all geodesics have length $\pi$, and the range of the sectional curvatures is $[1,4]$. The orbit space $M / G$ is then parametrized by $r \in\left[0, \frac{\pi}{2}\right]$, the volume of the orbits is given by a function $V(r)$ which vanishes at $r=0, \frac{\pi}{2}$, and the (unique) orbit of maximal volume is automatically minimaly imbedded in $M / G$, i.e. our equator. Let $q \in C(p)$ and let $H=G_{q}$ be the isotropy group of the $G$-action at $q$. The action of $H$ on $M$ then 
defines the cohomogeneity two transformation group which we will apply to prove Theorem 1-3.

\section{The orbital invariants of the isotropy action.}

Let $M=\mathbf{C P}(n), \mathbf{H} P(n)$, or $C a(2)$ and $p \in M$, let $G$ be the isometryisotropy subgroup at $p$. Let $X$ be a unit vector in $T_{p} M$, then the geodesic segment $\exp r X, r \in\left[0, \frac{\pi}{2}\right]$ parametrizes the orbit space $M / G$ and selects a representative triple of subgroups $K \subset H \subset G$ for the almost homogeneous $G$-space $M$, with $K=G_{\exp r X}, r \in\left(0, \frac{\pi}{2}\right)$ and $H=G_{q}, q=\exp \frac{\pi}{2} X \in C(p)$. We summarize the orbital geometry of the $G$-action and give explicit details for the case $M=C a(2)$.

Let $M=F / G$ as a symmetric space and $\underline{F}=\underline{G}+\underline{P}$ be the Car$\tan$ decomposition of the corresponding Lie algebras. For $C a(2)$ we have: $F=F_{4}, G=\operatorname{Spin} 9, \underline{P}$ is the spin representation $\Delta_{9}$ as a $G$-module, $K=\operatorname{Spin}^{-} 7, H=\operatorname{Spin} 8$, with corresponding orbit types:

$$
\begin{aligned}
& r=0:\{p\}, \\
& r \in\left(0, \frac{\pi}{2}\right): G / K=S_{r}, \quad \text { the geodesic sphere of radius } r, \\
& r=\frac{\pi}{2}: G / H=C(p) .
\end{aligned}
$$

Furthermore: $\Delta_{9} \mid H=\Delta_{8}^{+} \oplus \Delta_{8}^{-}$, the direct sum of the two half-spin representations, where $X \in \Delta_{8}^{+}$, and $\Delta_{8}^{+}\left|K=\rho_{7} \oplus \theta, \Delta_{8}^{-}\right| K=\Delta_{7}$ with $\Delta_{7}$ the spin representation, $\rho_{7}$ the standard orthogonal representation of $K=\operatorname{Spin}^{-} 7$, and $\theta_{X}$ the trivial one-dimensional representation along $X$. (Note: Here $\operatorname{Spin}^{-} 7$ is a non-standard imbedding of Spin 7 in Spin 9 , see the discussion in Section 3). For the isotropy action of $G$ on $T_{p} M$ the orbits are standard spheres of radius $r, S(r)$, where $\exp _{p} S(r)=S_{r}, r \in\left(0, \frac{\pi}{2}\right)$. Let furthermore $\underline{G}=\underline{H}+\rho_{8}^{\prime}$ and $\underline{H}=\underline{K}+\rho_{7}^{\prime}$ be orthogonal decompositions. Since $\rho_{8}^{\prime} \mid K=\Delta_{7}^{\prime}$, we may now write:

(i) $\quad \underline{G}=\underline{K}+\rho_{7}^{\prime}+\Delta_{7}^{\prime}$

(ii) $\quad \underline{F}_{4}=\underline{K}+\Delta_{7}^{\prime}+\rho_{7}^{\prime}+\rho_{7}+\theta_{X}+\Delta_{7}$

as $K$-modules (markings to distinguish different isomorphic submodules). By (i) we identify the tangent space $T_{K}(G / K)$ with $\Delta_{7}^{\prime}+\rho_{7}^{\prime}$; the resulting isomorphisms (needed later) between the tangent spaces of the $S_{r}^{\prime} s$, viewed as $\Delta_{7}^{\prime}+\rho_{7}^{\prime}$, and the orthogonal complement of $X$ in $T_{p} M$ are simultaneously realized in Proposition 3.

Definition. Define the map $J(r): \underline{G} \rightarrow T_{\exp r X} S_{r}$ by $J(r)(Z)=$ the Killing field of $Z$ at the point $\exp (r X) G, r \in\left(0, \frac{\pi}{2}\right)$. 
Proposition 3. Let $Z \in \underline{G}$ as above. Then we have:

(a) $J(0)(Z)=0, \frac{D}{d r} J(0)(Z)=[Z, X]$, Ker $J(r)=\underline{K}$, for $r \in\left(0, \frac{\pi}{2}\right)$.

(b) For $Z \in \Delta_{7}^{\prime} ; J(r)(Z)=(\sin r)(\operatorname{Exp} r X)_{\star}([Z, X])$. For $Z \in \rho_{7}^{\prime}$ $J(r)(Z)=\frac{1}{2}(\sin 2 r)(\operatorname{Exp} r X)_{\star}([Z, X])$.

(c) $\operatorname{ad} X \mid \Delta_{7}^{\prime}$ is an isomorphism onto $\Delta_{8}^{-} \cdot \operatorname{ad} X \mid \rho_{7}^{\prime}$ is an isomorphism onto the orthogonal complement of $X$ in $\Delta_{8}^{+}$.

(Left translation by $\exp r X$ has been denoted $\operatorname{Exp} r X$ ).

Proof. $\frac{D}{\partial r} \frac{\partial}{\partial t}(\exp t Z)(\exp r X) G=\frac{D}{\partial t} \frac{\partial}{\partial r} \exp (\operatorname{Ad}(\exp t Z)(r X)) G=[Z, X]$ at $r=t=0$. The result in (b) is well known from the solution of the Jacobi equation in rank one symmetric spaces. From $G$-invariance of the inner product it follows that $[\underline{G}, X] \subseteq X^{\perp}$, from two-point homogeneity it follows that $[\underline{G}, X]$ has codimension 1, i.e. $[\underline{G}, X]=X^{\perp}=\rho_{7}+\Delta_{7}$. Since $[\underline{H}, X] \subset$ $\Delta_{8}^{+}$and $[\underline{K}, X]=0,(\mathrm{c})$ now follows from (i) and (ii).

Corollary . The Riemannian homogeneous space $S_{r}$ is obtained from $G / K$ by the $K$-invariant inner product defined by the two scaling factors $\frac{1}{2} \sin 2 r$ and $\sin r$ on $\rho_{7}^{\prime}$ and $\Delta_{7}^{\prime}$ respectively, relative to the round unit sphere.

Remark 1. For the $G$-action on $T_{p} M$ we have of course the uniform scaling factor $r$ on $\rho_{7}^{\prime}+\Delta_{7}^{\prime}$ for $S(r)$, and an analogous statement Proposition 3'.

Remark 2. Any $G$-invariant metric on $G / K$ is defined by a $K$-invariant inner product on $\rho_{7}^{\prime}+\Delta_{7}^{\prime}$; by Schur's lemma any such inner product is defined by two scaling factors relative to the unit sphere.

\section{The orbital invariants of the cohomogeneity two action.}

We now proceed to the computation of the orbital invariants of the cohomogeneity two action of $H$ on $M$. Since the spheres $S_{r}$, viewed as homogeneous spaces $G / K$, are not in general naturally reductive, some complications may be expected.

We again specify for the case $M=C a(2)$, and first recall some of the needed facts about the relevant exceptional groups.

From the triality principle there are three distinct conjugacy classes of Spin 7 in Spin 8; they are conjugate under outer automorphisms of Spin 8 (arising from inner automorphisms of $F_{4}$ ). Two of these, denoted by $\mathrm{Spin}^{-} 7$ and $\operatorname{Spin}^{+} 7$ are conjugate in Spin 9 , with $\operatorname{Spin} 9 / \operatorname{Spin}^{ \pm} 7=S^{15}$. The last, denoted by $\operatorname{Spin}^{\times} 7$, is in standard position in Spin 9 , with $\operatorname{Spin} 9 / \operatorname{Spin}^{\times} 7$ equal to the Stiefel manifold of two-frames in $\mathbf{R}^{9}$. The intersection of two non-conjugate Spin 7 groups in $H=\operatorname{Spin} 8$ is a $G_{2}$, and Spin $7 / G_{2}=S^{7}$. The orbit space $T_{p} M / H \times H$ is the first quadrant $\mathbf{R}_{+}^{2}$, and the unit sphere $S(1)$ projects to the unit circle in $\mathbf{R}_{+}^{2}:\left\{e^{i t} \mid t \in\left[0 ; \frac{\pi}{2}\right]\right\}$. The orbit types for 
the $H \times H$-action on $T_{p} M=\Delta_{8}^{+}+\Delta_{8}^{-}$are:

$$
\begin{aligned}
& t=0: H \times H / \operatorname{Spin}^{-} 7 \times H=S^{7} \\
& t \in\left(0, \frac{\pi}{2}\right): H \times H / \operatorname{Spin}^{-} 7 \times \operatorname{Spin}^{+} 7=S^{7} \times S^{7} \\
& t=\frac{\pi}{2}: H \times H / H \times \operatorname{Spin}^{+} 7=S^{7} .
\end{aligned}
$$

Proposition 5. Let $\Delta$ be the diagonal imbedding of $H$ into $H \times H$. Then we have: The orbits of the restriction of the $H \times H$-action to either: (a) the $\operatorname{Spin}^{+} 7 \times \operatorname{Spin}^{-} 7$-action, or (b) the $\Delta H$-action, coincide with the full $H \times H$-orbits. $(\Delta H$ is the diagonal imbedding of $H$ into $H \times H)$.

Proof. Since $\operatorname{Spin}^{-} 7 \cap \operatorname{Spin}^{+} 7=G_{2}$, we have as principal orbit types: For (a): $\operatorname{Spin}^{+} 7 \times \operatorname{Spin}^{-} 7 / G_{2} \times G_{2} ;$ For (b): Spin $8 / G_{2}$; these have full dimension in the $H \times H$-orbits. The singular orbits are straightforward.

Proposition 6. Let $\underline{G}=\underline{K}+\rho_{7}^{\prime}+\Delta_{7}^{\prime}$ be the $K$-module decomposition of $\underline{G}$ as before, and let $Z$ be a unit vector in $\Delta_{7}^{\prime}$ with respect to the standard metric (scaling factors $(1,1)$ ). Then: (a) The orbit space $S_{r} / H$ is parametrized by $\exp (\theta Z) K, \theta \in\left[0, \frac{\pi}{2}\right]$, with the following orbit types:

$$
\begin{aligned}
& \theta=0: H_{K}=\operatorname{Spin}^{-} 7, H / H_{K}=S^{7} . \\
& \theta \in\left(0, \frac{\pi}{2}\right): H_{\operatorname{Exp}(t Z) K}=G_{2}, H / G_{2}=S^{7} \times S^{7} . \\
& \theta=\frac{\pi}{2}: H_{\operatorname{Exp}\left(\frac{\pi}{2} Z\right) K}=\operatorname{Spin}^{+} 7, H / \operatorname{Spin}^{+} 7=S^{7} .
\end{aligned}
$$

(b) $\operatorname{Exp}(\theta Z) K, \theta \in \mathbf{R}$, is a geodesic for any $G$-invariant metric on $G / K$.

Proof. Since (a) does not depend on the choice of metric, it suffices to prove it for the standard metric; i.e. for $S(1) \subseteq T_{p} M=\Delta_{8}^{+}+\Delta_{8}^{-}$as an $H$-module. This coincides with the $\Delta H$-module $T_{p} M$ considered in Proposition 5 (where the $H \times H$-action is the outer direct sum representation of $\Delta_{8}^{+}$and $\Delta_{8}^{-}$). Since $S(1)$ is a naturally reductive homogeneous space with respect to the pair $(K, G)$, it follows that $\operatorname{Exp}(t Z) K$ is a geodesic starting at $X \in \Delta_{8}^{+}$with velocity $Y=[Z, X]$. By Proposition $3^{\prime}, Y$ is a unit vector in $\Delta_{8}^{-}$, hence $\operatorname{Exp}\left(\frac{\pi}{2} Z\right) K \in \Delta_{8}^{-}$, and $\operatorname{Exp}(\theta Z) K$ projects bijectively onto $S(1) / H, \theta \in$ $\left[0, \frac{\pi}{2}\right]$. Moreover, $\Delta_{7}^{\prime}+\rho_{7}^{\prime} \mid G=\mu_{7}+\mu_{7}+\mathbf{R} Z$, where $\mu_{7}$ is the standard representation of $G_{2}$ on the pure Cayley numbers; hence the fixed point set of $G_{2}$ is $\{\operatorname{Exp}(\theta Z) K ; \theta \in \mathbf{R}\}$, which is then totally geodesic in any $G$ invariant metric.

Proposition 7. Let $\underline{F_{4}}=\underline{K}+\Delta_{7}^{\prime}+\rho_{7}^{\prime}+\rho_{7}+\theta_{X}+\Delta_{7}$ be the $K$-module decomposition of $\underline{F}_{4}$ as in $2(\mathrm{ii})$; and let $Z$ be a unit vector in $\Delta_{7}^{\prime}$ as above. 
Then $\gamma(\theta)=(\operatorname{Exp} \theta Z)(\operatorname{Exp} r X) G$ is a geodesic in $S_{r}$, orthogonal to the $H$ orbits.

Proof. By the previous proposition $\gamma(\theta)$ is a geodesic, by the Gauss lemma it is orthogonal to the $K$-orbits. Setting $Y=[Z, X]$ as above, we have from the proof of Proposition 6: $H_{\operatorname{Exp}\left(\frac{\pi}{2} Z\right)(\operatorname{Exp} r X) G}=\operatorname{Spin}^{+} 7$, hence, by the Gauss lemma applied to the point $\gamma\left(\frac{\pi}{2}\right), \gamma(\theta)$ is also orthogonal to the Spin ${ }^{+} 7$-orbits. The tangent spaces of the $\operatorname{Spin}^{-} 7$ and the $\operatorname{Spin}^{+} 7$-orbits together span the tangent spaces of the $H$-orbits.

Since $Z \in \Delta_{7}^{\prime}$, it follows from Proposition 3 that the $Y$-direction has scaling factor $\sin r$, hence the orbit space $H \backslash F / G$ is now parametrized by $(r, \theta) \in\left[0, \frac{\pi}{2}\right] \times\left[0, \frac{\pi}{2}\right]$ with the orbital distance metric $d s^{2}=d r^{2}+\sin ^{2} r d \theta^{2}$, i.e. a regular spherical triangle. This can be made more explicit as follows: $P=\exp _{p}(\mathbf{R} X+\mathbf{R} Y)$ is a real projective plane; as the fixed point set of $G_{2}$ it is totally geodesic in $M$; from the above discussion it must intersect all $H$-orbits perpendicularly. It is sufficient to identify $H$-orbits in the disk $D$ of radius $\frac{\pi}{2}$ in the 2 -plane $\mathbf{R} X+\mathbf{R} Y$ in $T_{p} M$. The centers $Z\left(\operatorname{Spin}^{-} 7\right)=\mathbf{Z}_{2}$ and $Z\left(\operatorname{Spin}^{+} 7\right)=\mathbf{Z}_{2}$ act on $F\left(G_{2}\right)$; here $Z\left(\operatorname{Spin}^{-} 7\right)$ acts as $-I d$ on $\Delta_{8}^{-}$and $Z\left(\operatorname{Spin}^{+} 7\right)$ acts as $-I d$ on $\Delta_{8}^{+}$. Reflections around the $X$ - and $Y$-axes then identify points on the same $H$-orbit, and it follows that the intersection of $D$ with the first quadrant may be used to parametrize the orbit space.

In the orbit space the corners are the fixed points of $H=\operatorname{Spin} 8$, the edges are the fixed point sets of three non-conjugate Spin 7's in $H$, permuted by t e triality automorphisms.

The volume functional of the cohomogeneity two action. The scaling factor which determines the relative volumes of the $G$-orbits $S_{r}$ is given by $\sin ^{8} r \sin ^{7} 2 r$. It remains to determine the scaling factors for the $H$-orbits $H \cdot(\exp \theta Z) K, \theta \in\left(0, \frac{\pi}{2}\right)$ in $S_{r}$. The Killing fields defined by $\underline{H}$ will again span the tangent spaces of the $H$-orbits, and hence determine the relative volumes. Although these Killing fields are well understood as vector fields (those corresponding to $\operatorname{Spin}^{-} 7$ and $\operatorname{Spin}^{+} 7$ are the standard Jacobi-fields on the sphere of $\sin \theta, \cos \theta$-type, respectively), their metric properties in terms of the $S_{r}$-metric are more complicated. The $K$-invariant decomposition $T_{K}(G / K)=\rho_{7}^{\prime} \oplus \Delta_{7}^{\prime}$ defines a $G$-invariant decomposition of the tangent bundle $T(G / K)$ as a Whitney sum of two subbundles. However, it does not follow from this that a Killing field defined by $Z \in \underline{K}$ remains in the $\Delta_{7}^{\prime}$ summand of scaling factor $\sin r$ (the Killing field is given by transvections along $(\exp \theta Z) K$ with respect to the standard metric, i.e. those transvections are in $S O\left(T_{p} M\right)=S 0(16)$, and need not respect the above splitting of $T(G / K))$. Since we are only interested in the volume element determined by those Killing fields, the result turns out to be easy. 
Proposition 8. Let $\exp (\theta Z) K$ be a geodesic in $S_{r}$ with $Z$ a unit vector in $\Delta_{7}^{\prime}$ as before. Then the scaling factor for the relative volumes of the orbits $H \exp (\theta Z) K, \theta \in\left(0, \frac{\pi}{2}\right)$ is given by $\sin ^{7} \theta \cos ^{7} \theta$.

Proof. Since $\operatorname{Exp}(-\theta Z)_{\star}$ is an isometry with respect to any $G$-invariant metric on $S_{r}$, the Killing fields pull back to vectors in $T_{K}(G / K)$ of length $\sin \theta$ and $\cos \theta$, respectively, with respect to the standard metric. Since the orthogonal complements of $Z$ coincide for all $K$-invariant inner products on $\rho_{7}^{\prime}+\Delta_{7}^{\prime}$, the result follows.

Corollary. The volume functional for the $H$-action on $M$ is given by $V(r, \theta)=\sin ^{7} \theta \cos ^{7} \theta \sin ^{7} r \sin ^{7} 2 r$.

Proof. Since $Z \in \Delta_{7}^{\prime}$ corresponds to a factor $\sin r$, and the volume of $S_{r}$ is $\sin ^{8} r \sin ^{7} 2 r$, it follows that the $r$-dependance of $V(r, \theta)$ must be $\sin ^{7} r \sin ^{7} 2 r$.

Remark. This is the same result as would be obtained by formal lifting computations in a hypothetical "Hopf fibration" $S^{7} \rightarrow S^{23} \rightarrow \mathrm{Ca}(2)$.

We summarize the corresponding results for the remaining cases:

Complex projective space. $\mathbf{C} P(n)=U(n+1) / U(n) \times U(1), G=U(n) \times$ $U(1), H=U(n-1) \times U(1) \times U(1)$. The normal subgroup $G^{1}=U(n)$ and $H^{1}=G^{1} \cap H=U(n-1) \times U(1)$ have the same orbits as $G$ and $H$ respectively, - hence we study their actions. We have $K=U(n-1)$ and the $K$-module $\underline{P}=\mu_{n-1} \oplus 2 \theta$, where $\mu_{n-1}$ is the standard representation of $U(n-1)$ and $\theta$ the trivial representation. The scaling factors $\sin r$ and $\frac{1}{2} \sin 2 r$, defining the metrics of the Berger-spheres $S_{r}^{2 n-1}$, have multiplicities $2 n-2$ and 1 , respectively. The principal $H$-orbits are of the type $S^{2 n-3} \times S^{1}$, and the volume functional is given by $\sin ^{2 n-3} r \sin 2 r \sin ^{2 n-3} \theta \cos \theta$.

Quaternionic projective space. $H P(n)=S p(n+1) / S p(n) \times S p(1), G=$ $S p(n) \times S p(1), H=S p(n-1) \times S p(1) \times S p(1)$. Again we can reduce to the normal subgroup $S p(n)$ and $S p(n-1) \times S p(1)$. We have: $K=S p(n-1)$, the $K$-module $\underline{P}=\nu_{n-1}+4 \theta$, where $\nu_{n-1}$ is the standard representation of $S p(n-1)$. The scaling factors $\sin r$ and $\frac{1}{2} \sin 2 r$ now have multiplicities $4 n-4$ and 3, repectively. The principal $H$-orbits are of type $S^{4 n-5} \times S^{3}$ and the volume functional is given by $\sin ^{4 n-5} r \sin ^{3} 2 r \sin ^{4 n-5} \theta \cos ^{3} \theta$.

Change of coordinates. For convenience we define new spherical coordinates by changing the center to the corner $\left(r=0, \theta=\frac{\pi}{2}\right)$. (Except for $n=2$, this is not an $H$-fixpoint.) In these coordinates the volume functionals now become: $\sin ^{2} r \cos ^{2 n-3} r \sin 2 \theta$ and $\sin ^{6} r \cos ^{4 n-5} r \sin ^{3} 2 \theta$ for $\mathbf{C P}(n)$ and $\mathbf{H} P(n)$, respectively (for $C a(2)$ there is no change). 


\section{The analysis and geometry of the reduced differential equation.}

The previous results can be summarized as follows:

Let $M$ be a projective space of complex, quaternionic, or Cayley-type. Let $H$ be the isometry group that fixes a pair of conjugate points in $M$. Then the following holds:

1. The orbit space $M / H$ with the orbital distance metric is a regular spherical triangle parameterized by $(r, \theta) \in\left[0, \frac{\pi}{2}\right] \times\left[0, \frac{\pi}{2}\right] ; d s^{2}=d r^{2}+$ $\sin ^{2} r d \theta^{2}$.

2. The volumes of the $H$-orbits are given by the functional $V(r, \theta)=$ $\sin ^{2 b} r \cos ^{a} r \sin ^{b} 2 \theta$, where:

$$
\begin{aligned}
& a=2 n-3, b=1 \text { for } \mathbf{C} P(n), \\
& a=4 n-5, b=3 \text { for } \mathbf{H} P(n), \\
& a=b=7 \text { for } C a(2) .
\end{aligned}
$$

Let $\gamma(s)=(r(s), \theta(s))$ be a curve in the interior of $M / H$, parameterized by arc length. By Proposition 2 the mean curvature of the corresponding hypersurface of $M$ is given by $k(\gamma(s))-\frac{d}{d \bar{n}} \ln V$, where the geodesic curvature $k=\dot{\alpha}+\dot{\theta} \cos r$, and $\bar{n}=-\sin \alpha \frac{\partial}{\partial r}+\cos \alpha \sin ^{-1} r \frac{\partial}{\partial \theta} ; \alpha$ is the angle from $\frac{\partial}{\partial r}$ to the tangent vector $\dot{\gamma}(s)$. Straightforward computation now gives the following result:

Proposition 9. The hypersurface corresponding to the curve $\gamma(s)=(r(s)$, $\theta(s))$ is minimal if and only if the curve $\Gamma(S)=(r(s), \theta(s), \alpha(s))$ satisfies the following dynamical system:

$$
\begin{aligned}
\dot{r} & =\cos \alpha \\
\dot{\theta} & =\sin \alpha \sin ^{-1} r \\
\dot{\alpha} & =K_{r} \sin \alpha+2 b \cos \alpha \sin ^{-1} r \cot 2 \theta,
\end{aligned}
$$

where $K_{r}=a \tan r-(2 b+1) \cot r$.

This is singular along the edges $\theta=0, \frac{\pi}{2}$ and $r=\frac{\pi}{2}$; by well known arguments ([T2]) there exists, for each interior point $P$ of an edge, a unique solution $\gamma(s)$ with initial value at $P$. This solution is analytic and has initial direction orthogonal to the edge; thus it defines a smooth minimal hypersurface in $M$. Solution curves with end point at the origin are more complicated, we will need the following observation:

Proposition 10. Let $\Gamma(s)=(r(s), \theta(s), \alpha(s))$ be a solution of $(*)$ with 
$\lim _{s \rightarrow 0^{-}} r(s)=0$; assume that the number of $\theta$-critical point of $\Gamma(s)$ is bounded for $s \in(-\delta, 0)$. Then $\Gamma(s)$ is the solution curve $\theta \equiv \frac{\pi}{4}$.

Proof. a) Since $\dot{\theta}=\sin \alpha \sin ^{-1} r$, it follows that $\sin \alpha$ has constant sign in $(-\epsilon, 0)$ for some positive $\epsilon$. Since $\ddot{r}$ at an $r$-critical point is easily computed to be $-\sin ^{2} \alpha K_{r}$, it follows that $r$-critical points must be minima for $r<$ $r_{0}=\operatorname{Arctan} \sqrt{(2 b+1) a^{-1}}$ and maxima for $r>r_{0}$. Hence, from the condition $\lim r(s)=0$, we may also assume $\cos \alpha<0$ in $(-\epsilon, 0)$.

b) Similarly, a computation of $\ddot{\alpha}$ at a critical point for $\alpha$ gives: $\sin \alpha\left(a \cos ^{-2}\right.$ $\left.r \cos \alpha+(2 b+1) \sin ^{-2} r \cos \alpha\right)-2 b \cos \alpha \sin ^{-2} r \tan ^{-2} 2 \theta(\cos r \cos \alpha \tan 2 \theta-$ $2 \sin \alpha \cos ^{-2} 2 \theta$ ), where both terms have the same constant sign in some $(-\epsilon, 0)$. Here the sign of $\tan 2 \theta$ is found by plugging back into the condition $\dot{\alpha}=0$, i.e. $\tan 2 \theta=-2 b \cos \alpha \sin ^{-1} r K_{r}^{-1} \sin ^{-1} \alpha$. Hence, $\dot{\alpha}$ must have constant sign in some interval $(-\epsilon, 0)$; and $\alpha_{1}=\lim _{s \rightarrow 0-} \alpha(s)$ exists.

c) We prove that $\alpha_{1}=\pi$ and $\theta_{1}=\frac{\pi}{4}$. We have: $\frac{d \theta}{d r}=\sin ^{-1} r \tan \alpha \approx$ $\sin ^{-1} r \tan \alpha_{1}$ for small $\epsilon$, i.e. $\theta(r) \approx C+\frac{1}{2}\left(\tan \alpha_{1}\right) \ln \left[(1-\cos r)(1+\cos r)^{-1}\right]$ $\underset{r \rightarrow 0+}{\longrightarrow} \infty$ unless $\tan \alpha_{1}=0$; hence $\alpha_{1}=\pi$ follows. Also, $\frac{d \alpha}{d r}=K_{r} \tan \alpha+$ $2 b \sin ^{-1} r \cot 2 \theta \approx 2 b \cot 2 \theta_{1} \sin ^{-1} r$ if $\cot 2 \theta_{1} \neq 0$. By a similar integration this would imply $|\alpha(r)| \underset{r \rightarrow 0-}{\rightarrow} \infty$, which is a contradiction. Hence $\theta_{1}=\frac{\pi}{4}$, and the above solution curve must be tangent to $\theta \equiv \frac{\pi}{4}$ at the origin.

d) To conclude the proof, assume that $\sin \alpha>0$ in $(-\epsilon, o)$, hence $\theta(s)<$ $\frac{\pi}{4}$ in $(-\epsilon, 0)$. From $(*)$ this implies that $\dot{\alpha}$ becomes negative as soon as $(2 b+1) \cot r \sin \alpha$ dominates $a \tan r \sin \alpha$; this contradicts $\lim _{s \rightarrow 0-} \alpha(s)=\pi$ unless $\alpha \equiv \pi, \theta \equiv \frac{\pi}{4}$. The case $\sin \alpha<0$ is treated similarly.

Definition. We define the following points in $M / G: O=(0,0), A=$ $\left(\frac{\pi}{2}, 0\right), \quad B=\left(\frac{\pi}{2}, \frac{\pi}{2}\right), \quad C=\left(\frac{\pi}{2}, \frac{\pi}{4}\right), \quad D=\left(r_{0}, 0\right), \quad E=\left(r_{0}, \frac{\pi}{2}\right)$, with $r_{0}=$ $\operatorname{Arctan} \sqrt{(2 b+1) a^{-1}}$.

Proposition 11. The following curves define solutions of $(*)$ :

$$
\begin{aligned}
& r \equiv r_{0}=\operatorname{Arctan} \sqrt{(2 b+1) a^{-1}} \\
& \theta \equiv \frac{\pi}{4} \\
& \sin r \sin \theta \equiv \sqrt{b(a+2 b+1)^{-1}} \\
& \sin r \cos \theta \equiv \sqrt{b(a+2 b+1)^{-1}} .
\end{aligned}
$$

Proof. We have $\alpha \equiv \pm \frac{\pi}{2}$ for (i), $\alpha \equiv 0$ or $\pi$ for (ii), and the result follows directly by substitution into (*). The solutions (iii) and (iv) correspond to 
the orbits of maximal volume for the isotropy groups of the points $B$ and $A$, respectively.

Remark. (i), (iii) and (iv) define smooth hypersurfaces of $M$, here (iii) and (iv) are "equators", i.e. homogeneous, minimal hyperspheres. Similarly, any solution curve of $(*)$ with initial point on the interior of the edge $O A$ and terminal point on the interior of $A B$ must define a smooth, minimal hypersphere in $M$. The most effective technique so far for proving existence of solution curves which are "closed" in the generalized sense that they have end points on the singular boundary, is to study variations of the pattern of critical points of auxilary functions along solution curves.

Definition. Let $\Gamma(s)=(r(s), \theta(s), \alpha(s))$ be a solution curve for $(*)$, $s \geq 0$. Let $r_{m}^{i}(\gamma)$ and $r_{M}^{i}(\gamma)$ denote the $i$-th local minimum, respectively local maximum, for $r(s), s>0$; similarly for $\theta_{m}^{i}(\gamma)$ and $\theta_{M}^{i}(\gamma)$.

Proposition 12. Let $\Gamma(s)=(r(s), \theta(s), \alpha(s)), s \geq 0$, be a solution curve for (*) in $X^{*}=M^{*} / H$, which does not coincide with (i) or (ii). Then the critical points of $r(s)$ and $\theta(s)$ are non-degenerate, and no two of them can coincide. Moreover, we have: Any $r_{m}^{i}(\gamma)$ occurs with $r<r_{0}, r_{M}^{i}(\gamma)$ with $r>r_{0}$. Any $\theta_{m}^{i}(\gamma)$ occurs with $\theta<\frac{\pi}{4}, \theta_{M}^{i}(\gamma)$ with $\theta>\frac{\pi}{4}$.

Proof. From $(*)$ we have $\ddot{r}=-(a \tan r-(2 b+1) \cot r)$ at a critical point for $r$, hence $\ddot{r} \neq 0$ unless $r=r_{0}$; by the uniqueness theorem for differential equations a solution with $r\left(s_{0}\right)=r_{0}, \cos \alpha\left(s_{0}\right)=0$ must be (i). Similarly, a critical point for $\theta$ is non-degenerate unless $\theta=\frac{\pi}{4}$ and $\sin \alpha=0$, i.e. the solution (ii). At an $r$-critical point we have $\sin \alpha= \pm 1$, hence $\dot{\theta} \neq 0$. Straightforward computation of $\ddot{r}$ and $\ddot{\theta}$ concludes the proof.

Definition. The $(r, \theta)$-pattern $P(\Gamma)$ of the above curve $\Gamma(s)$ is the following sequence: Assign symbols $r_{M}^{i}, r_{m}^{i}, \theta_{M}^{i}, \theta_{m}^{i}$ in the same order as corresponding critical points occur along $\Gamma(s) ; s>0$. At a boundary point critical points of $r$ and $\theta$ coincide, in that case the pair is enclosed in a bracket and considered as one symbol. $P^{N}(\Gamma)$ is the segment consisting of the $N$ first symbols of $P(\Gamma)$.

Definition. Let $\Gamma_{d}(s)=\left(r_{d}(s), \theta_{d}(s), \alpha_{d}(s)\right)$ denote the solution curve with $r_{d}(0)=\frac{\pi}{2}, \theta_{d}(0)=d, \alpha_{d}(0)=-\pi$, and let $\gamma_{d}(s)=\left(r_{d}(s), \theta_{d}(s)\right)$.

We need the following observation:

Proposition 13. The curve $\gamma_{d}$ depends continuously on $d \in\left(0, \frac{\pi}{2}\right)$.

Proof. This is the "continuous dependance of solutions beyond intersections with the singular boundary"; for proof see $[\mathbf{H T}]$. Notice that also $\Gamma_{d}$ depends 
continuously on initial conditions modulo identification of $\alpha$ with $\alpha+\pi$ at boundary points.

Proposition 14. Let $I=\left(b_{1}-\epsilon, b_{1}+\epsilon\right) \subseteq\left(0, \frac{\pi}{2}\right)$. Assume that $P^{N-1}\left(\Gamma_{b}\right)$ has no symbol of bracket type for $b \in I$ and that $P^{N}\left(\Gamma_{b_{1}}\right)$ has $N$-th symbol of bracket type. Then we have:

(1) $P^{N-1}\left(\Gamma_{d}\right)$ is constant for $d \in I$.

(2) Let $p$ be the first intersection point of $\Gamma_{b_{1}}(s)$ with the boundary $(s>0)$; the $N$-th symbol of $P^{N}\left(\Gamma_{b_{1}}\right)$ is then as follows:

$$
\begin{aligned}
& p \in O D:\left(r_{m}^{i}, \theta_{m}^{j}\right) \\
& p \in O E:\left(r_{m}^{i}, \theta_{M}^{j}\right) \\
& p \in D A \text { or } A C:\left(r_{M}^{i}, \theta_{m}^{j}\right) \\
& p \in E B \text { or } B C:\left(r_{M}^{i}, \theta_{M}^{j}\right) .
\end{aligned}
$$

(3) $P^{2 N}\left(\Gamma_{b_{1}}\right)$ is reflectionally symmetric about its $N$-th symbol, and $P\left(\Gamma_{b_{1}}\right)$ is periodic with period $2 N$.

Proof. This follows from Proposition 12, a careful analysis of the sharp turning of solution curves near the singular boundary, estimates on $\dot{\alpha}$ from $(*)$, and Proposition 13. (For detailed arguments of this type, we refer to [T1].) For (3), just observe that if $\gamma_{b_{1}}\left(s_{0}\right)$ is the first boundary intersection point, then the solution curve $\gamma_{b_{1}}(s), s \in\left[o, s_{0}\right]$, can be extended to $\left[0,2 s_{0}\right]$ by retracing backwards, and then extended to all $s$ by periodicity.

Remark. In contrast to $P^{N}(\Gamma)$, the whole pattern $P(\Gamma)$ will not, in general, be constant on any open interval.

We now turn to the most delicate analysis that is needed: the initial part of $P\left(\Gamma_{d}\right)$ as $d \rightarrow \frac{\pi}{4}$; this is determined by the intersection pattern of $\gamma_{d}(s)$ with the solution $\theta \equiv \frac{\pi}{4}$.

Let $\bar{\theta}=\theta-\frac{\pi}{4}$, straightforward computation from $(*)$ gives:

$$
\text { (**) } \begin{aligned}
& \frac{d^{2} \bar{\theta}}{d r^{2}}+\left[\cot r-\cos ^{-2} \alpha(a \tan r-(2 b+1) \cot r)\right] \frac{d \bar{\theta}}{d r} \\
&++2 b \sin ^{-2} r \cos ^{-2} \alpha \tan 2 \bar{\theta}=0 .
\end{aligned}
$$

By continuous dependance of solution curves on initial conditions it follows that on compact intervals contained in $\left[0, \frac{\pi}{2}\right), \alpha_{d} \rightarrow-\pi . \bar{\theta}_{d} \rightarrow 0$ as $d \rightarrow \frac{-\pi}{4}$. We can then approximate by the linearization of $\left(^{* *}\right)$ along the solution $\bar{\theta} \equiv 0$ :

$(* * \prime) \quad \frac{d^{2} \bar{\theta}}{d r^{2}}+[2(b+1) \cot r-a \tan r] \frac{d \bar{\theta}}{d r}+4 b \bar{\theta} \sin ^{-2} r=0$. 
The substitution $\bar{\theta}=v \cos ^{-\frac{a}{2}} r \sin ^{-(b+1)} r$ gives:

$(* * *)$

$$
\frac{d^{2} v}{d r^{2}}+q(r) v=0, v\left(\frac{\pi}{2}\right)=0
$$

where $q(r)=\frac{a}{2}(2 b+3)+5 b+1+\left(3 b-b^{2}\right) \cot ^{2} r-\frac{a(a-2)}{4} \tan ^{2} r$. As $r \rightarrow 0+$, the dominating term is $b(3-b) \cot ^{2} r$, and we have:

For $\mathbf{C P}(n): b=1 ; 2 \cot ^{2} r \rightarrow_{r \rightarrow 0+} \infty$

For $\mathbf{H} P(n): b=3: 0$

For $C a(2): b=7:-28 \cot ^{2} r \rightarrow_{r \rightarrow 0+}-\infty$.

Hence the oscillatory behaviour near $r=0$ of $\gamma_{d}$ as $d \rightarrow \frac{\pi}{4}$ changes drastically with the scalar field: the term forces rapid oscillations in the $\mathbf{C P}(n)$-case, it counteracts oscillations in the $C a(2)$-case, $\mathbf{H} P(n)$ must be intvestigated separately.

We estimate the number of zeroes of a non-zero solution of $(* * *)$ on the interval $[\epsilon, T]$ by applying a delicate criterion based on Sturm's comparison method:

Proposition 15. Let $I=[\epsilon, T], \epsilon>0, q(r) \geq 0$ for $r \in I$, and let $N$ be the number of zeroes of a non-zero solution of the equation $\left(^{* * *}\right)$ on $I$. Then we have: $\left|\pi N-\int_{\epsilon}^{T} \sqrt{q(r)} d r\right| \leq \pi+\frac{1}{4} \int_{\epsilon}^{T} \frac{|d q(r)|}{q(r)}$.

Proof. This is given in [Ha], Th 5-2, p. 347 .

Proposition 16. Let $M=\mathbf{C} P(n)$; i.e. $a=2 n-3, b=1$, and $\delta \in\left(0, \frac{\pi}{2}\right)$. Then any solution of $\left({ }^{* *}\right)$ has infinitely many zeroes on $(0, \delta)$.

Proof. For $\delta$ sufficiently small we have $q(r)>p(r)=1,9 \cot ^{2} r$ and it suffices to consider $v^{\prime \prime}+p(r) v=0$. Here $\int_{\epsilon}^{\delta} \sqrt{1.9 \cot ^{2} r} d r=\sqrt{1.9}(\ln (\sin \delta)-$ $\ln (\sin \epsilon))$, and $\frac{1}{4} \int_{\epsilon}^{\delta}|d \ln p(r)|=-\frac{1}{2} \ln (\cot \epsilon)+\frac{1}{2} \ln (\cot \delta)$, hence the difference approaches infinity as $\epsilon \rightarrow 0+$, and the result follows from Proposition 15 .

Theorem 1. There exists infinitely many congruence classes of imbedded, minimal hyperspheres in $\mathbf{C} P(n), n \geq 2$.

Proof. Let $P_{m}\left(\Gamma_{d}\right)$ be the segment of the $(r, \theta)$-pattern of $\Gamma_{d}$ up to and including the first $r_{m}$. From Proposition 16 it follows that the number of intersections of the curve $\gamma_{d}(s), s \in\left[0, \frac{\pi}{2}\right]$ with $\theta \equiv \frac{\pi}{4}$ approaches infinity as $d \rightarrow \frac{\pi}{4}$. Hence $P_{m}\left(\Gamma_{d}\right)$ becomes a $\theta_{m}, \theta_{M}$-sequence of length $N$ terminated by an $r_{m}$, where $N \rightarrow \infty$ as $d \rightarrow \frac{\pi}{4}$. By Proposition 14 it follows that as $d \rightarrow \frac{\pi}{4}$, it passes through an infinite number of values $d^{\prime}$ such that $\gamma_{d^{\prime}}$ intersects the singular boundary before the first $r_{m}$. By Proposition 10 this intersection 
occurs in the interior of one of the edges $O A$ or $O B$; by Proposition 11 $\gamma_{d^{\prime}}$ determines a minimal hypersphere in $M$. Since $\gamma_{d^{\prime}}$ does not have selfintersections before the first $r_{m}$ this hypersphere is imbedded.

For $\mathbf{H} P(n)$ and $C a(2)$ it is not possible to obtain the desired solutions from the local oscillation properties around 0 . It is needed to obtain long-time control of the solution curves emanating from the boundary $A B$ to find the intersections with $O A$ and $O B$. The most delicate analytical result required for this is given by the following:

Proposition 17. Let $M=\mathbf{H} P(n)$ or $C a(2)$. Then there exists a positive $\delta$ such that for $d \in\left(\frac{\pi}{4}, \frac{\pi}{4}+\delta\right)$ the solution curve $\gamma_{d}(s)$ intersects $\theta \equiv \frac{\pi}{4}$ twice before $r_{m}^{1}\left(\gamma_{d}\right)$.

Proof. We prove this for the $\mathbf{H} P(n)$ case, and refer the $C a(2)$-case to the Appendix.

From Proposition 15 and standard approximation arguments it follows that it is sufficient to show that:

(A) $\int_{\epsilon}^{T} \sqrt{q(r) d r}>2 \pi+\frac{1}{4} \int_{\epsilon}^{T} \frac{|d q(r)|}{q(r)}$ for some positive $\epsilon$. To minimize the computations, we reduce to a previously treated case as follows: The results in [HS], imply that $Q(r)=\frac{(2 d+1) m-4 d}{2}-\frac{(m-2)(m-4 d)}{4} \tan ^{2} r$ satisfies (A), (for some $\left.T \in\left(0, \frac{\pi}{2}\right)\right)$, when $d=3, m \geq 12$. It is clear that if (A) holds for some $Q(r)$, it also holds for $\bar{Q}(r)=C_{1} Q(r)+C_{2}$, where $C_{1} \geq 1, C_{2} \geq 0$. Now choose $C_{1}=(4 n-5)(4 n-7)(m-2)^{-1}(m-12)^{-1}, \quad C_{2}=18 n-$ $\frac{13}{2}-\frac{1}{2}(7 m-12)(4 n-5)(4 n-7)(m-2)^{-1}(m-12)^{-1}$ and $d=3$. Then $C_{1} Q(r)+C_{2}=18 n-\frac{13}{2}-\frac{1}{4}(4 n-5)(4 n-7) \tan ^{2} r=q(r)$. It remains only to observe that choosing $m=4 n$ makes $C_{1} \geq 1, C_{2} \geq 0$.

Corollary. There exists a $d \in\left(\frac{\pi}{4}, \operatorname{Arccos} \sqrt{b(a+2 b+1)^{-1}}\right)$ such that $P\left(\Gamma_{d}\right)$ is periodic with period $\left(\theta_{m}, r_{m}, \theta_{M},\left(\theta_{m}, r_{M}\right), \theta_{M}, r_{m}, \theta_{m},\left(\theta_{M}, r_{M}\right)\right)$.

Proof. The solution curve (iv) of Propostion 11 has periodic $(r, \theta)$-pattern with period $\left(\left(\theta_{m}, r_{m}\right),\left(\theta_{M}, r_{M}\right)\right)$. Hence it follows from Propositions 13 that for $d$ close to Arccos $\sqrt{b(a+2 b+1)^{-1}}$ the second intersection point of $\gamma_{d}$ with $\theta \equiv \frac{\pi}{4}$ occurs after $r_{m}^{1}\left(\gamma_{d}\right)$, with $\alpha \in\left(0, \frac{\pi}{2}\right)$. By Proposition 16 and continuous dependence on initial conditions, it follows that for $d \in\left(\frac{\pi}{4}, \frac{\pi}{4}+\delta\right)$ with sufficiently small $\delta$, the second intersection point of $\gamma_{d}$ with $\theta \equiv \frac{\pi}{4}$ has $\alpha \in\left(\frac{\pi}{2}, \pi\right)$. Hence, by continuity, $\alpha=\frac{\pi}{2}$ at the second intersection point of $\gamma_{d}$ with $\theta \equiv \frac{\pi}{4}$ for some $d \in\left(\frac{\pi}{4}+\delta, \operatorname{Arccos} \sqrt{b(a+2 b+1)^{-1}}\right)$. This is then the first $r_{m}$, and the solution is continued through reflection around $\theta \equiv \frac{\pi}{4}$, to yield the above periodic $(r, \theta)$-pattern. 
Remark. Computer pictures of solutions close to this one are shown in the appendix.

Proposition 18. There are infinitely many $d \in\left(\frac{\pi}{4}, \frac{\pi}{2}\right)$ such that $\gamma_{d}$ intersects the boundary arc $O D$.

Proof. Let $d_{1}=\operatorname{Arccos} \sqrt{b(a+2 b+1)^{-1}}$. Since $P\left(\Gamma_{d_{1}}\right)$ is periodic with period $\left(\left(r_{m}^{1}, \theta_{m}^{1}\right),\left(r_{M}^{1}, \theta_{M}^{1}\right)\right)$, it follows from Proposition 13 that for $d$ sufficiently close to $d_{1}$, the number of $\theta_{m}$ 's before $r_{m}^{2 N}$ in $P\left(\Gamma_{d}\right)$ is either $2 N-1$ or $2 N$. Let $\bar{d}$ be as in the preceding corollary, with the period of $P\left(\Gamma_{\bar{d}}\right)$ equal to $\left(\theta_{m}, r_{m}, \theta_{M},\left(\theta_{m}, r_{M}\right), \theta_{M}, r_{m}, \theta_{m},\left(\theta_{M}, r_{M}\right)\right)$. Again, by Proposition 13 , the number of $\theta_{m}$ 's before $r_{m}^{2 N}$ in $P\left(\Gamma_{d}\right)$ is now $3 N-1$ when $d$ is sufficiently close to $\bar{d}$. It follows form Proposition 14 that as $d$ passes through the interval $\left(\frac{\pi}{4}, \operatorname{Arccos} \sqrt{b(a+2 b+1)^{-1}}\right), \gamma_{d}$ must have intersected $O D$ at last $N-1$ times.

Theorem 2. There exists infinitely many congruence classes of immersed minimal hyperspheres in $\mathbf{H} P(n), n \geq 1$.

Theorem 3. There exists infinitely many congruence classes of immersed, minimal hyperspheres in the Cayley projective plane, Ca(2).

Proof. These theorems are now corollaries of Proposition 18 and Proposition 11.

\section{Appendix.}

Estimates for the Cayley projective plane. In this case we have:

$$
\begin{aligned}
(* * *) \quad \frac{d^{2} v}{d r^{2}}+q(r) v=0, v\left(\frac{\pi}{2}\right) & =0, \\
& \text { with } q(r)=95.5-28 \cot ^{2} r-8,75 \tan ^{2} r .
\end{aligned}
$$

This time the now negative term $b(3-b) \cot ^{2} r$ limits the region where $\left(^{* * *}\right)$ is of oscillating type, i.e. $q(r)>0$, to $\left[r_{1}, r_{2}\right]$, where $r_{1} \approx 0,50216, r_{2} \approx$ 1,27296. Here $\int_{r_{1}}^{r_{2}} \sqrt{q(r) d r}<2 \pi$, so there is no hope of applying Proposition 15 to prove Proposition 17, as was done for $\mathbf{H} P(n)$. In that case an argument was needed that applied for all $n$. Now, however, we need only the case $n=2$, i.e. the above equation $\left({ }^{* *}\right)$; which opens for brute force estimates. Computer pictures of solution curves close to those of Proposition 17 and its corollary are given at the end. Let $w=-\frac{1}{v} \frac{d v}{d r}$, we then have:

$$
\frac{d w}{d r}=q+w^{2} .
$$


Recalling that $v=\bar{\theta} \cos ^{\frac{7}{2}} r \sin ^{8} r$, it is clear that crossings with $\bar{\theta} \equiv 0$ corresponds to zeroes of $v$, i.e. to $w$-crossings with $\pm \infty$ (notice that we are in the region where $\alpha \neq \pm \frac{\pi}{2}$, i.e. $\frac{d v}{d r} \neq 0$ ). To obtain suitable bounds for $w$, we observe the following:

Lemma. Let $w(r)$ and $\bar{w}(r)$ be solutions of $\frac{d w}{d r}=q+w^{2}$ and $\frac{d \bar{w}}{d r}=C+$ $\bar{w}^{2}$ respectively, defined on the interval $\left[r^{\prime}, r^{\prime \prime}\right]$, and assume that $q \geq C$ on $\left[r^{\prime}, r^{\prime \prime}\right], \bar{w}\left(r^{\prime \prime}\right)>w\left(r^{\prime \prime}\right)$. Then $\bar{w}>w$ on $\left[r^{\prime}, r^{\prime \prime}\right]$.

Proof. Notice that $\frac{d}{d r}(w-\bar{w})=q-C+w^{2}-\bar{w}^{2}$ is positive whenever $w=\bar{w}$; this contradicts the possibility of the two solutions crossing on $\left[r^{\prime}, r^{\prime \prime}\right]$.

The solution of $\frac{d \bar{w}}{d r}=-A^{2}+\bar{w}^{2}$ with initial value $\bar{w}\left(r^{\prime}\right)=B$ is given by:

(A1): $A\left(e^{2 A\left(r^{\prime}-r\right)}(B+A)+B-A\right)\left(e^{2 A\left(r^{\prime}-r\right)}(B+A)-(B-A)\right)^{-1}$.

The special case $\lim _{r \rightarrow r^{\prime}} \bar{w}(r)=\infty$ by:

(A2): $A\left(e^{2 A\left(r^{\prime}-r\right)}+1\right)\left(e^{2 A\left(r^{\prime}-r\right)}-1\right)^{-1}$.

The solution of $\frac{d \bar{w}}{d r}=A^{2}+\bar{w}^{2}$ with initial value $\bar{w}\left(r^{\prime}\right)=B$ is given by:

(A3): $A \tan \left(A\left(r-r^{\prime}\right)+\operatorname{Arctan} \frac{B}{A}\right)$.

The special case $\lim _{r \rightarrow r^{\prime}} \bar{w}(r)=\infty$ by:

(A4): $A \tan \left(A\left(r-r^{\prime}\right)+\frac{\pi}{2}\right)$.

We note that $q(r)$ reaches its maximum at $r_{3} \approx 0,92878$; is increasing in $\left(0, r_{3}\right)$ and decreasing in $\left(r_{3}, \frac{\pi}{2}\right)$. We will obtain relevant bounds as follows:

(i) For $\left[r^{\prime}, r^{\prime \prime}\right] \subset\left(r_{2}, \frac{\pi}{2}\right)$ : The comparison equation is $\frac{d \bar{w}}{d r}=-A^{2}+\bar{w}^{2}$ with $A^{2} \geq-q\left(r^{\prime \prime}\right)$.

(ii) For $\left[r^{\prime}, r^{\prime \prime}\right] \subset\left(r_{3}, r_{2}\right)$. The comparison equation is $\frac{d \bar{w}}{d r}=A^{2}+\bar{w}^{2}$, with $A^{2} \leq \inf _{r \in\left[r^{\prime}, r^{\prime \prime}\right]} q(r)$, i.e. $A^{2} \leq q\left(r^{\prime \prime}\right)$.

(iii) For $\left[r^{\prime}, r^{\prime \prime}\right] \subset\left(r_{1}, r_{3}\right)$ the comparison equation is as in (ii) with $A^{2} \leq$ $q\left(r^{\prime}\right)$.

(iv) For $\left[r^{\prime}, r^{\prime \prime}\right] \subset\left(0, r_{1}\right)$ : The comparison equation is as in (i) with $A^{2} \geq$ $-q\left(r^{\prime}\right)$.

We have $\lim _{r \rightarrow \frac{\pi}{2}-} w(r)=\lim _{r \rightarrow \frac{\pi}{2}-} \frac{d}{d r} \ln v(r)=\infty$, and we wish to bound $w(r)$ from above as $r$ decreases from $\frac{\pi}{2}$. We give the steps in detail, the numerical values can now readily be checked on a calculator.

a) We choose to start with the interval $\left[1,55 ; \frac{\pi}{2}-0,01\right]$, and apply (i) with $A^{2}=87398,7>q\left(\frac{\pi}{2}-0,01\right)$; since we have no upper estimate for $w\left(\frac{\pi}{2}-0,01\right)$, we choose the case $\bar{w}\left(\frac{\pi}{2}-0,01\right)=\infty$. By (A2) we get: $\bar{w}(1,55)=$ $A\left(e^{2 A\left(\frac{\pi}{2}-1,56\right)}+1\right)\left(e^{2 A\left(\frac{\pi}{2}-1,56\right)}-1\right)^{-1}<296,7$. 
b) We divide $(1.30,1.55)$ into intervals $\left(r^{\prime}, r^{\prime \prime}\right)$ of length at most 0.02 , and apply (i), case (A1), where the initial value $B$ is set equal to the previously obtained upper bound for $w\left(r^{\prime \prime}\right)$. At each step, care is taken in rounding off to insure the validity of the upper bound. This gives $w(1.30)<10,77$; a final application of this procedure to $[1,273,1.30]$ gives $w(1,273)<8,78$.

c) We divide $(0,95 ; 1,272)$ into intervals of length at most 0,01 and apply (ii), (A3). This gives the estimate $\bar{w}(0.95)<-28,5$. Let $\left[r^{\prime}, r^{\prime \prime}\right]=$ $[0,915 ; 0,95]$, then $\inf _{r \in\left[r^{\prime}, r^{\prime \prime}\right]} q(r)=q(0.95)>64,07=A^{2}$. We can then again apply (ii), (A3), and obtain: $\frac{B}{A}<-\frac{28,5}{8,005}, \operatorname{Arctan} \frac{B}{A}<-1,2969, A$. $(0,915-0,95)<-0,28$; hence the argument of $\tan$ in (A3) has already passed $-\frac{\pi}{2}$; hence $\bar{w}(r)$, and, by comparison, $w(r)$, has passed $-\infty$ before $r$ reaches 0.915 .

Corollary. The first zero of $v(r)$, as $r$ decreases from $\frac{\pi}{2}$, occurs for $r>$ 0.915 .

d) On the interval $[0,91 ; 0,915]$ we apply (iii), (A4), to obtain $w(0,91)<$ $\bar{w}(0,91)<200$.

e) Dividing $(0,52 ; 0,91)$ into intervals of length 0,01 , and applying (iii), case (A3), we obtain the bound $w(0,52)<-10,8$. Continuing with 3 intervals of length 0,005 , we obtain: $w(0,505)<-12,8$.

g) It follows that $w(0,50)<-12,8$; we are now in the region $q<0$. Applying (iv), (A1) to the intervals $[0,48 ; 0,50]$ and $[0,45 ; 0,48]$, we obtain $w(0,45)<-32,4$. Now consider the denominator $D(r)=\left(e^{2 A(0,45-r)}(B+\right.$ $A)-(B-A))$ of $(\mathrm{A} 1)$ on $[0,40 ; 0,45]$, with $A^{2}=62,71>-q(0,4), B=$ $-32,4$. Then $D(0,45)=2 A>0 . D(0,40) \approx-13,7<0$, hence $D(r)=0$ for some $r \in(0,40 ; 0,45)$, this implies $\bar{w}(r)$, and hence $w(r)$ reaches $-\infty$ in this interval.

Corollary. The solution $v(r)$ of $\left({ }^{* * *}\right)$ has at least two zeroes on the interval $0,40<r<\frac{\pi}{2}$. This completes the proof of Proposition 17 for the Ca(2)-case.

The computer pictures of solutions shown on the next pages were obtained in collaboration with Dr. Gil Bor. 
Solution of $(*)$ for $a=7, b=3,: H P(3)$.

The two solutions shown are for $d-\frac{\pi}{4}=0.06$ and 0.07 , respectively.

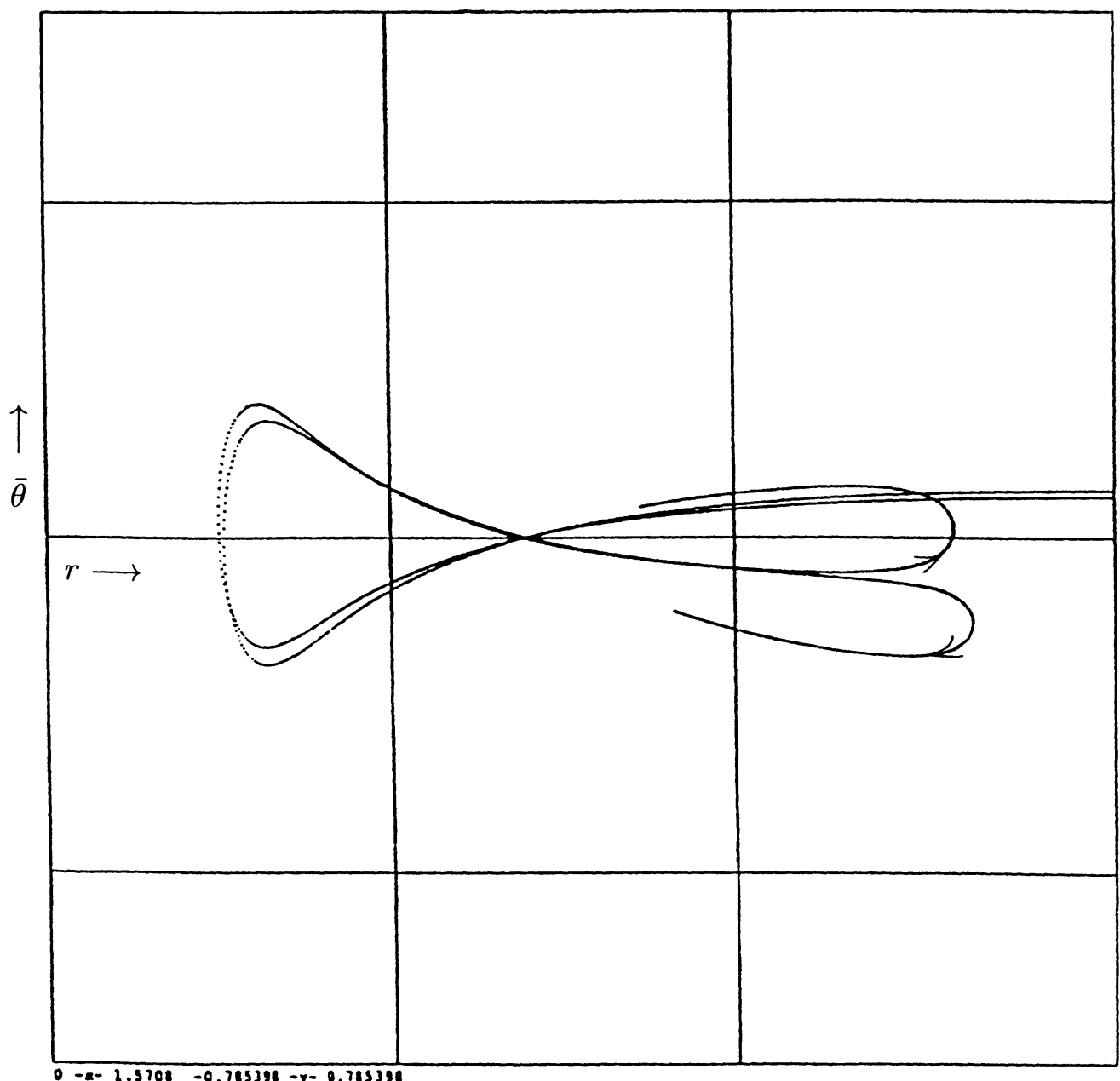


Solution of $(*)$ for $a=7, b=7,: C a(2)$.

The two solutions shown are for $d-\frac{\pi}{4}=0.6,0.4 ; 0.2 ; 0.1 ;$ and 0.05 . Touching with the boundary occurs for a value between 0.2 and 0.1 .

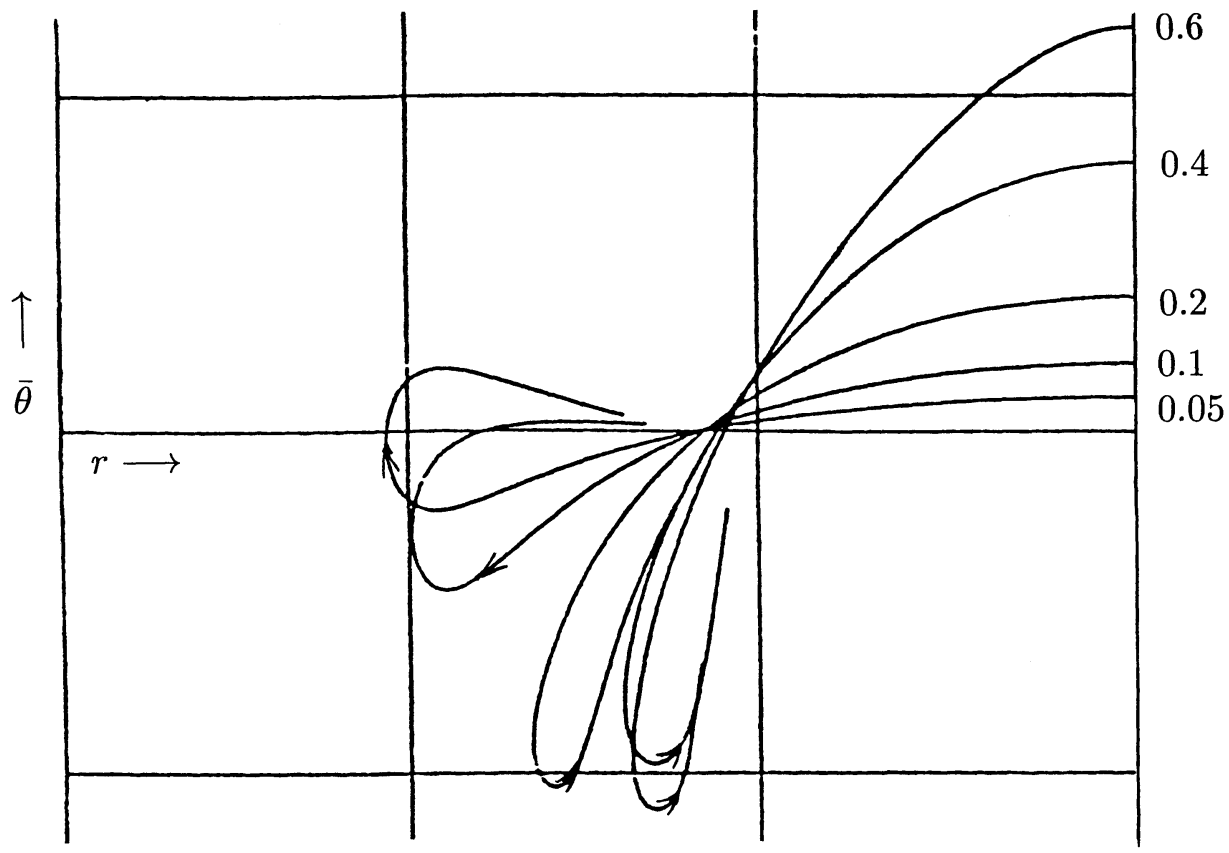

Solution of $(*)$ for $a=7, b=7,: C a(2)$.

The two solutions shown are for $d-\frac{\pi}{4}=0.04$ and 0.05 , respectively.

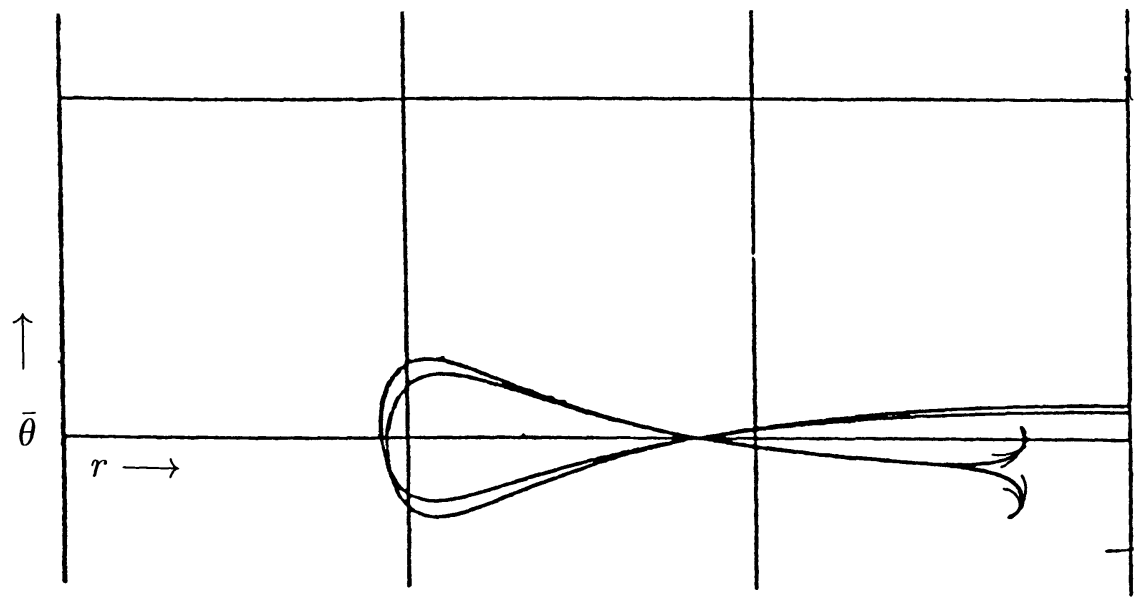




\section{References}

[F] T. Frankel, On the fundamental group of a minimal submanifold, Annals of Math., 83 (1966), 68-73.

[FK] D. Ferus and H. Karcher, Non-rotational minimal spheres and minimal cones, Comment. Math. Helv., 60 (1985), 247-269.

[Ha] P. Hartmann, Ordinary Differential Equations, N. Y. Wiley. (1964).

[H1] W.Y. Hsiang, Minimal cones and the spherical Bernstein problem I, Annals of Math., 118 (1983), 63-71.

[H2] Minimal cones and the spherical Bernstein problem II, Invent. Math., 74 (1983), 351-369.

[HHT-1] W.T. Hsiang, W.Y. Hsiang and P. Tomter, On the construction of infinitely many, mutually non-congruent examples of minimal imbeddings of $S^{2 n-1}$ into $\mathbf{C} P^{n}, n \geq 2$, Bull. A.M.S., 8 (1983), 463-465.

[HHT-2] - On the existence of minimal hyperspheres in compact symmetric spaces, Ann. Scient Ec. Norm. Sup., 21 (1988), 287-305.

[HL] W.T. Hsiang and I. Sterling, Minimal cones and the spherical Bernstein problem III, Invent. Math., 85 (1986), 223-247.

[HS] W.Y. Hsiang and H.B. Lawson, Minimal submanifolds of low cohomogeneity, J. Diff. Geom., 5 (1970), 1-37.

[HT] W.Y. Hsiang and P. Tomter, On minimal immersions of $S^{n-1}$ into $S^{n}(1), n \geq 4$, Ann. Scient. Ec. Norm. Sup., 20 (1987), 201-214.

[KN] S. Kobayashi and K. Nomizu, Foundations of differential geometry, Vol. 1-2, N. Y. Interscience.

[T1] P. Tomter, The spherical Bernstein problem in even dimensions and related problems, Acta Math., 158 (1987), 187-212.

[T2] - Existence and uniqueness for a class of Cauchy problems with characteristic initial manifolds, J. Diff. Equations, 71 (1988), 1-9.

Received March 6, 1993, revised May 17, 1993.

UNIVERSITY OF OSLO

OSLO, NORWAY 


\title{
PACIFIC JOURNAL OF MATHEMATICS
}

Founded by

\author{
$\begin{array}{ll}\text { E. F. Beckenbach (1906-1982) F. Wolf (1904-1989) } & \text { F }\end{array}$
}

EDITORS

Sun-Yung A. Chang (Managing Editor) Robert Finn

University of California

Los Angeles, CA 90095-1555

pacific@math.ucla.edu

F. Michael Christ

University of California

Los Angeles, CA 90095-1555

christ@math.ucla.edu

Nicholas Ercolani

University of Arizona

Tucson, AZ 85721

ercolani@math.arizona.edu
Stanford University

Stanford, CA 94305

finn@gauss.stanford.edu

Steven Kerckhoff

Stanford University

Stanford, CA 94305

spk@gauss.stanford.edu

Martin Scharlemann

University of California

Santa Barbara, CA 93106

mgscharl@math.ucsb.edu
Gang Tian

Massachusettes Institute of Technology

Cambridge, MA 02139

tian@math.mit.edu

\section{S. Varadarajan}

University of California

Los Angeles, CA 90095-1555

vsv@math.ucla.edu

Dan Voiculescu

University of California

Berkeley, CA 94720

dvv@math.berkeley.edu

\section{SUPPORTING INSTITUTIONS}

\author{
CALIFORNIA INSTITUTE OF TECHNOLOGY \\ NEW MEXICO STATE UNIVERSITY \\ OREGON STATE UNIVERSITY \\ STANFORD UNIVERSITY \\ UNIVERSITY OF ARIZONA \\ UNIVERSITY OF BRITISH COLUMBIA \\ UNIVERSITY OF CALIFORNIA \\ UNIVERSITY OF HAWAII
}

\author{
UNIVERSITY OF MONTANA \\ UNIVERSITY OF NEVADA, RENO \\ UNIVERSITY OF OREGON \\ UNIVERSITY OF SOUTHERN CALIFORNIA \\ UNIVERSITY OF UTAH \\ UNIVERSITY OF WASHINGTON \\ WASHINGTON STATE UNIVERSITY
}

The supporting Institutions listed above contribute to the cost of publication of this Journal, but they are not owners or publishers and have no responsibility for its contents or policies.

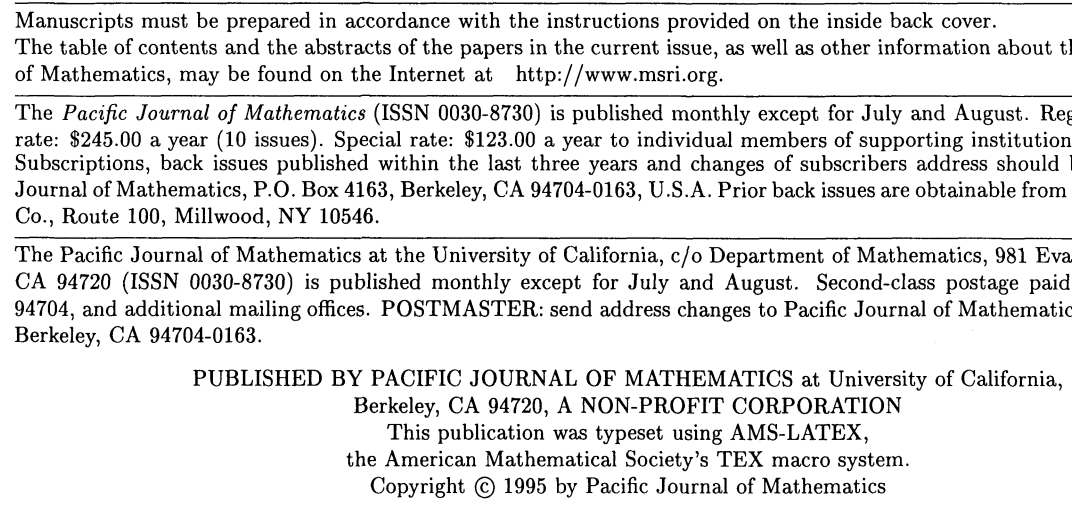




\section{PACIFIC JOURNAL OF MATHEMATICS}

\section{Volume $173 \quad$ No. $1 \quad$ March 1996}

Isometric immersions of $H_{1}^{n}$ into $H_{1}^{n+1}$

KINETSU ABE

Rotationally symmetric hypersurfaces with prescribed mean curvature

MARIE-FRANÇOISE BIDAUT-VÉRON

The covers of a Noetherian module

JIAN-JUN CHUAI

On the odd primary cohomology of higher projective planes

MARK FoSKEY and MiCHAEL DAVID SLACK

Unit indices of some imaginary composite quadratic fields. II

MiKinito HiRABAYASHI

Mixed automorphic vector bundles on Shimura varieties

MIN Ho LeE

Trace ideal criteria for Toeplitz and Hankel operators on the weighted Bergman

spaces with exponential type weights

PENG LIN and RICHARD ROCHBERG

On quadratic reciprocity over function fields

KATHY DONOVAN MERRILL and LYNNE WALLING

$\left(A_{2}\right)$-conditions and Carleson inequalities in Bergman spaces

TAKAHIKO NAKAZI and MASAHIRO YAMADA

A note on a paper of E. Boasso and A. Larotonda: "A spectral theory for solvable Lie 173 algebras of operators"
C. Отт

Tensor products with anisotropic principal series representations of free groups

Carlo Pensavalle and Tim Steger

On Ricci deformation of a Riemannian metric on manifold with boundary

YING SHEN

The Weyl quantization of Poisson $S U(2)$

Albert Jeu-Liang Sheu

Weyl's law for $S L(3, \mathbb{Z}) \backslash S L(3, \mathbb{R}) / S O(3, \mathbb{R})$

ERIC GeORge Stade and Dorothy IRENe Wallace (ANDREOli)

Minimal hyperspheres in two-point homogeneous spaces

PER TOMTER

Subalgebras of little Lipschitz algebras

NiKOLAI ISAAC WEAVER 